\title{
Teorias de enfermagem na promoção do conforto em dermatologia
}

\author{
Theories of nursing in promotion of comfort in dermatology \\ Teorías de enfermería en la promoción del confort en dermatología
}

Euzeli da Silva Brandão'; Iraci dos Santos"

\begin{abstract}
RESUMO
Objetivo: refletir sobre a aplicação dos conceitos de Henderson e Kolcaba durante o cuidado a pessoa com afecção cutânea, visando à promoção do conforto, além da autonomia do enfermeiro. Conteúdo: estudo de reflexão pautado nos conceitos de Henderson, Kolcaba e nos atributos para avaliação do conforto em dermatologia. A restauração do equilíbrio das dimensões física, mental e espiritual exige uma fundamentação filosófica, teórica e tecnológica. Esta encontra respaldo na filosofia de Henderson, que visualizava a função da enfermeira como específica, autônoma e independente. A existência de necessidades de conforto não atendidas pressupõe a necessidade de intervenção. Assim, utilizou-se a análise conceptual do termo conforto, proposto por Kolcaba, incluindo três sentidos técnicos e quatro contextos, além dos atributos como: dor, mobilidade, padrão de sono, entre outros, considerados essenciais na avaliação do conforto em dermatologia. Conclusão: esta reflexão poderá contribuir para utilização das teorias citadas na prática de cuidar em dermatologia.
\end{abstract}

Descritores: Enfermagem; dermatologia; teoria de enfermagem; conforto do paciente.

\section{ABSTRACT}

Objective: to think about application of the concepts of Henderson and Kolcaba during care for people with cutaneous conditions, with a view to affording comfort, besides autonomy for the nurses involved. Content: this reflective study was based on the concepts of Henderson and Kolcaba and on attributes for the evaluation of comfort in dermatology. Restoration of balance in the physical, mental and spiritual dimensions requires a philosophical, theoretical and technological foundation. This is supported by Henderson's philosophy, which viewed the nurse's role as specific, autonomous, and independent. The existence of unmet need for comfort presupposes the need for intervention. Accordingly, the term comfort proposed by Kolcaba, including three technical senses and four contexts, was analyzed conceptually, as were attributes considered essential in assessing comfort in dermatology, such as pain, mobility, sleep pattern, and others. Conclusion: this reflection may contribute to the use of the theories cited in practical care in dermatology.

Descriptors: Nursing; dermatology; nursing theory; patient comfort.

\section{RESUMEN}

Objetivo: reflexionar sobre la aplicación de los conceptos de Henderson y Kolcaba durante el cuidado a la persona con afección cutánea, buscando la promoción del confort, además de la autonomía del enfermero. Contenido: estudio de reflexión basado en los conceptos de Henderson, Kolcaba y en los atributos para evaluación del confort en dermatología. La restauración del equilibrio de las dimensiones física, mental y espiritual exige una fundamentación filosófica, teórica y tecnológica. Esta encuentra respaldo en la filosofía de Henderson, que visualizaba la función de la enfermera como específica, autónoma e independiente. La existencia de necesidades de confort no atendidas presupone la necesidad de intervención. Siendo así, se utilizó el análisis conceptual del término confort, propuesto por Kolcaba, incluyendo tres sentidos técnicos y cuatro contextos, además de los atributos como: dolor, movilidad, patrón de sueño, entre otros, considerados esenciales en la evaluación del confort en dermatología. Conclusión: esa reflexión podrá contribuir a la utilización de las teorías mencionadas en la práctica de cuidar en dermatología.

Descriptores: Enfermería; dermatología; teoría de enfermería; confort del paciente.

\section{INTRODUÇÃO}

A enfermagem é uma profissão que possui funções próprias e específicas para o desenvolvimento das práticas de cuidar, educar, gerenciar e pesquisar, visando à qualidade do atendimento de saúde junto à pessoa hospitalizada, em acompanhamento ambulatorial, em domicílios e comunidades. Entende-se que tais práticas vão além do cumprimento das prescrições médicas, incluindo, a avaliação da pessoa e implementação de cuidados que visam a abordagem na sua totalidade, e consequentemente a promoção do seu conforto. 
O desempenho das práticas citadas, tendo em vista a restauração do equilíbrio das dimensões física, mental e espiritual das pessoas, em especial das acometidas pelas afecções cutâneas, exige uma fundamentação filosófica, teórica e tecnológica que propicie autonomia e independência ao enfermeiro, levando-o às inovações características de um enfrentamento para uma enfermagem pós-moderna e futura conforme recomenda Jean Watson ${ }^{1}$.

Esta fundamentação encontra respaldo na filosofia de Virgínia Henderson, autora que visualizava a função da enfermeira como específica, autônoma e independente. Entre suas suposições destaca-se que uma definição de enfermagem deveria abarcar o princípio do equilíbrio fisiológico. Baseando-se na medicina psicossomática e suas implicações para a enfermagem, Henderson considera que o equilíbrio emocional é inseparável do equilíbrio fisiológico ${ }^{2}$.

Referente à autonomia e independência, é necessário compreender o enfermeiro como elemento central na vida da pessoa que necessita de cuidados. Trata-se de um profissional que, além de possuir funções próprias, garante o cumprimento das prescrições da equipe de saúde. Assim, as ações do enfermeiro e dos demais profissionais de saúde se superpõem².

Nesse sentido, este estudo teve por objetivo refletir sobre a aplicação dos conceitos de Virgínia Henderson e de Katharine Kolcaba durante o atendimento à pessoa com afecção cutânea, visando à promoção do conforto, além da autonomia do enfermeiro no desempenho de suas atividades.

\section{CONTEÚDO}

Sobre os conceitos de pessoa, sociedade/ambiente, saúde e enfermagem concebido por Henderson, destaca-se que eles revelam a necessidade da aquisição de competência para entender a diversidade cultural das pessoas e habilidades específicas para desenvolver a prática do cuidado individualizado ${ }^{3}$, inclusive a pessoa com afecção cutânea.

Assim, a pessoa deve ser vista como um ser integral, com componentes fisiológicos, psicológicos, sociológicos e espirituais inseparáveis, considerando a pessoa e sua família uma unidade. A sociedade/ambiente se caracteriza por um conjunto de todas as condições e influências exteriores que afetam a vida e o desenvolvimento. A saúde reflete a independência do indivíduo para realização de suas necessidades básicas.

Sobre a enfermagem, ressalta-se que a enfermeira possui como função auxiliar o indivíduo, na realização de atividades que ele realizaria se tivesse força, vontade ou conhecimentos necessários, de tal forma que lhe promova a sua independência ${ }^{3}$.

Nesse sentido, vale lembrar que Hildegard Peplau questionava: o que sabem as enfermeiras e como usam estes conhecimentos para ajudar as pessoas ${ }^{4}$ ?

\section{O conceito de conforto na Teoria de Katharine Kolcaba}

$\mathrm{Na}$ enfermagem, o uso do conceito de conforto foi encontrado no livro de Florence Nightingale Notas de Enfermagem (1859), evidenciando que esta autora já reconhecia o conforto como essencial ${ }^{5}$.

Em 1908, Aikens destacou que nada relacionado ao conforto é pequeno o suficiente para ser ignorado. Uma boa enfermeira é aquela que torna as pessoas confortáveis, sendo a promoção do conforto o fator principal para determinar a sua habilidade. Percebe-se que, naquela época, o conforto já era altamente valorizado, pois ampliava a possibilidade de cura, sendo resultado de intervenções de ordem física, emocional e ambiental ${ }^{5}$.

Porém, o significado e a importância do conforto passaram por mudanças consideráveis, tendo em vista a evolução dos cuidados com a saúde. Do seu significado geral, o conforto tinha um valor significativo para a enfermagem no início do século, passando para um valor menos importante e com uma conotação mais específica ao sentido físico. Para exemplificar, em 1950, com a maior popularidade dos analgésicos para controle da dor, poucas ações para promoção do conforto foram descritas 5 .

Na década de 70, as enfermeiras adquiriram maior autonomia e puderam implementar medidas de conforto sem as ordens médicas. No entanto, com a intensificação da tecnologia dura, muitas medidas de conforto tradicionais foram consideradas simples e relegadas a menor importância. O conforto não era mais considerado um foco da enfermagem especializada. O termo ainda estava indefinido na disciplina e semânticamente diversificado, com interpretação restrita, escrito raramente, e, claro, não mensurado 5 .

Na década de 80, ocorreram muitos avanços na medicina e a cura tornou-se mais provável através da cirurgia, dos antibióticos, da radiação e da quimioterapia. Narcóticos passaram a ser usados para a dor severa. Assim, o conforto tornou-se uma estratégia secundária em relação ao propósito de cura. Destaca-se que nessa década, a promoção do conforto era considerada uma meta menor, quando não havia mais a possibilidade de cura ${ }^{5}$. 
A conexão na literatura de enfermagem entre o conforto da pessoa para a reabilitação foi sutil, mas constante ao longo da história da enfermagem. As sementes da Teoria do conforto surgiram no início da década de 80 , dez anos depois, tal conceito na área de enfermagem foi ampliado e promovido em novas formas 5 .

Katharine Kolcaba começa a elaborar o conceito de conforto em 1982, contando com as contribuições de Janet Morse, Patricia Benner; Eloita Neves-Arruda (enfermeira brasileria), Larson e Afaf Meleis, entre outras; e, em 1992, operacionaliza o conceito 5 .

A existência de necessidades de conforto não atendidas pressupõe a necessidade de intervenção, ratificando a relação entre conforto e cuidado, e a preocupação em estabelecer medidas de conforto como sinônimo de intervenção de enfermagem ${ }^{6}$.

Nesse sentido, ressalte-se a análise conceptual do termo conforto, realizado por Kolcaba ${ }^{5-7}$. Para ela, conforto e cuidados de conforto são conceitos complexos, individuais e holísticos, um estado desejável, representando uma condição individual, relaxada, saudável e pacífica ${ }^{5}$.

Para Kolcaba conforto é uma experiência imediata de ter atendidas as necessidades humanas básicas, possuindo três sentidos técnicos: relief - alívio, easy - calma, e transcendence - transcendência. Para ela, as necessidades de conforto podem ser experenciadas em quatro contextos: físico, psicoespiritual, ambiental e sociocultural ${ }^{5-7}$.

\section{Os quatro contextos de Kolcaba e os atributos para avaliação do conforto em dermatologia}

Durante uma pesquisa que tinha como um dos objetivos analisar as evidências do cuidado de enfermagem mediante uma Tecnologia de Cuidados de Enfermagem a ser aplicada em pessoas com afecções cutâneas foi necessário determinar atributos considerados relevantes para o reconhecimento do padrão de (des)conforto ${ }^{8}$. Nesse sentido, foi considerada não somente a experiência da pesquisadora, mas também, o fato de ser especialista na área de dermatologia. Baseando-se no exposto e na representação de expansibilidade na determinação do (des)conforto da pessoa com afecção cutânea, a pesquisadora estabeleceu os atributos dor; mobilidade; padrão de sono; exposição do corpo e das lesões; conhecimento sobre a doença e autocuidado.

\section{Conforto físico: atributos dor, mobilidade e padrão de sono}

Relacionado às sensações do corpo, Kolcaba sabia que a dimensão fisiológica inerente aos fatores que afetam o estado físico era o contexto mais óbvio e consensual de conforto ${ }^{5}$.

O primeiro tema relacionava-se as necessidades de conforto no que diz respeito a doença, sendo o de trato mais prevalente o alívio da dor5. Por se tratar de uma experiência subjetiva e pessoal associada a dano real ou potencial nos tecidos, como, por exemplo, na pele, a dor é caracterizada por uma experiência multidimensional, podendo variar na intensidade sensorial, além de sofrer interferências afetivo-motivacionais. Descrita como quinto sinal vital, a dor aguda ou crônica deve ser avaliada e registrada desde a admissão ${ }^{9}$.

A avaliação da dor favorece o conhecimento de sua origem, considerando fatores emocionais, motivacionais, cognitivos e até mesmo de personalidade. Porém, devido à sua subjetividade, inexiste um instrumento padrão para sua mensuração objetiva. Entre os métodos disponíveis existem os que a avaliam somente a sua intensidade, considerados unidimensionais, como escalas de categoria numérica/verbal e analógico-visual e os que avaliam além dos fatores sensoriais, fatores afetivo-emocionais, sendo considerados multidimensionais ${ }^{9}$.

Por se tratar de uma das mais relevantes causas do sofrimento, a dor compromete a qualidade de vida das pessoas, provoca incapacidades, além de sérias repercussões psicossociais e econômicas10. Diante das características das afecções cutâneas, considera-se essencial estabelecer a dor como um atributo na avaliação do (des)conforto da pessoa acometida antes e após intervenções de enfermagem.

Uma visão adicional sobre o conforto físico aborda o posicionamento, destacando a necessidade de sentar corretamente, ter liberdade para se movimentar, de forma independente e ser capaz de voltar para a cama. Especificamente em relação as pessoas com afecções cutâneas, tal fato nos remete a importância de valorizar o atributo mobilidade.

A mobilidade é um dos aspectos da vida que contribui para a autovalorização e conforto, sendo utilizada para diferentes e múltiplos propósitos como satisfazer necessidades, realizar atividades básicas, recreativas, sociais, entre outras $^{11}$.

Por outro lado, a imobilidade pode ser responsável por alterações comportamentais variadas, como hostilidade, medo, ansiedade, além de afetar a capacidade de enfrentamento, podendo chegar a um quadro de depressão ${ }^{11}$.

As lesões cutâneas podem afetar consideravelmente a mobilidade em diferentes graus. Além disso, destaca-se a necessidade de movimentar a pessoa com segurança, evitando o aumento das áreas lesadas, incluindo o risco 
aumentado para desenvolvimento de lesões por pressão, atribuído não somente à dificuldade de mudança de decúbito, mas também a existência de outros fatores de risco preexistentes.

A presença de lesões cutâneas extensas e disseminadas pode contribuir para a redução da mobilidade, tendo em vista que as lesões ressecam, causando dor durante movimentação dos membros, além de ficarem aderidas nas superfícies de contato, como roupas de uso pessoal e de cama, causando dor durante o deslocamento. Tal fato pode fazer com que a pessoa permaneça em igual posição durante longos períodos, podendo causar alterações no ciclo sono vigília, caracterizando outro fator a ser discutido no próximo atributo estabelecido: padrão de sono.

O repouso está relacionado ao ato de sentar-se ou deitar-se confortavelmente em uma cama, momento em que as pessoas sentem-se relaxadas mentalmente, livres de ansiedade e fisicamente calmas. Ao descansar, apresentam um estado de atividade mental, física e espiritual que promove a renovação ${ }^{12}$.

Qualquer doença que cause dor, desconforto físico ou ansiedade e depressão resulta frequentemente em transtornos do sono ${ }^{12}$. Assim, quando de forma inesperada as pessoas se deparam com o adoecimento cutâneo, que trazem sérias repercussões físicas, emocionais e sociais, são fortemente afetadas em seu padrão de sono e repouso, fato que inevitavelmente pode agravar a doença. Além da dor física causada pelas lesões cutâneas, as pessoas apresentam preocupações reais, como as limitações físicas, o caráter desfigurante das lesões, o medo do desconhecido, o medo de não conseguir retornar ao seu estilo de vida habitual, entre outros que impedem o seu relaxamento.

\section{O conforto psicoespiritual e o atributo exposição do corpo e das lesões}

Um dilema que Kolcaba encontrou em sua revisão de literatura sobre holismo foi diferenciar as experiências da mente daquelas do espírito e das emoções. Assim, formou o contexto psicoespiritual, agregando os componentes mental, emocional e espiritual. A definição de conforto psicoespiritual refere-se à conscientização interna do eu, incluindo autoestima, autoconceito, sexualidade e significado na vida do indivíduo e ao seu relacionamento com uma ordem superior de $\operatorname{ser}^{5-7}$. Nesse sentido, ressalta-se a impossibilidade das pessoas com afecções cutâneas de omitir da sociedade um problema de saúde, pois está impresso na pele, causando a inevitável exposição das lesões.

O significado da mudança na aparência para o indivíduo é influenciado pela percepção de mudança e importância atribuída à imagem corporal. A exposição das lesões pode promover curiosidade, repugnância, medo, entre outros sentimentos normalmente perceptíveis na maneira de agir e de olhar ${ }^{13}$. A esse respeito cita-se que o problema da pessoa estigmatizada pode ser percebido simplesmente pelo ato de dirigir-lhe atenção, pois o fato de estar presente entre os "normais" a expõe cruelmente a invasões de privacidade ${ }^{14}$.

Nesse sentido, destaca-se que a forma como o ser humano se sente acerca dele mesmo é algo que afeta crucialmente todos os aspectos da sua experiência de vida, refletindo na capacidade de entender e dominar os problemas, defender os próprios direitos e necessidades ${ }^{15}$.

Esta situação pode provocar na pessoa com afecção cutânea sentimentos de tristeza, ansiedade, vergonha, fato que interfere diretamente na sua autoestima e consequentemente no seu conforto, afetando consequentemente as relações sociais, de trabalho, familiar e até mesmo com profissionais de saúde, quando despreparados para atender a esta clientela.

Ciente das inevitáveis influências do comprometimento da autoimagem e consequente exposição do corpo e das lesões, considera-se a tal exposição um atributo de extrema relevância na avaliação do conforto das pessoas com afecções cutâneas.

\section{Conforto ambiental}

Um ambiente adequado foi considerado importante para promoção da saúde e da cura pelos enfermeiros interessados em cuidar da pessoa de forma holística. A definição de conforto ambiental relaciona-se às condições e influências externas, ou seja, dizem respeito a fatores como ruído, cor, luz, temperatura, vista de janelas, acesso à natureza e aos elementos natural versus sintético ${ }^{5-7}$.

A esse respeito, destaca-se a escassez de enfermarias específicas destinadas aos clientes com afecções dermatológicas, e, consequentemente, com recursos estruturais para atendimento dessa clientela. Tal fato, os expõem ainda mais à estigmatização e aos riscos de infecção e infestação.

\section{Conforto sociocultural e o atributo conhecimento sobre a doença e o autocuidado}

$\mathrm{O}$ conceito de conforto sociocultural refere-se às relações interpessoais, familiares e sociais, inclusive relações financeiras, educacionais e de apoio. A idéia de cultura foi adicionada, para incluir histórias familiares, tradições, língua, uso de roupas e costumes, aspectos considerados importantes e facilitadores para a equipe de saúde, durante a internação hospitalar, para aumentar o conforto social ${ }^{5-7}$. 
Diante do início geralmente insidioso e quadro desfigurante das afecções cutâneas, as pessoas necessitam obter conhecimentos básicos que esclareçam o processo de adoecimento e que facilitem a promoção do autocuidado. A esse respeito, ressalta-se a importância de considerar o nível de conhecimento do próprio cliente sobre a doença/autocuidado, ou seja, a percepção dele em relação ao seu conhecimento.

Diante de tais considerações, a partir da compreensão do conforto como resultado dos cuidados de enfermagem tem-se as proposições da teoria que podem ser divididas em três momentos ${ }^{5}$ :

no primeiro momento, o enfermeiro avalia a pessoa de forma holística, integral, identificando as necessidades de conforto, a partir dos quatro contextos. Paralelamente, implementa intervenções, avaliando o conforto proporcionado pelas mesmas;

no segundo momento, as atividades que promovem conforto são intensificadas e a pessoa é estimulada a desenvolver comportamentos para promoção do seu conforto, que podem ser internos (cura, função imune...), externos (atividades de saúde), ou morte tranquila;

o terceiro momento corresponde à integridade institucional, quando instituição e equipe de cuidados são preparadas para aperfeiçoar a qualidade dos serviços, o que resulta na satisfação da pessoa, na redução de custos e de morbidade e reinternações, assim como melhores políticas e práticas de saúde.

\section{CONCLUSÃO}

A ideia de estudar as teorias de enfermagem apropriadas à promoção do conforto levou as autoras a iniciar com a Filosofia de Henderson, a qual considera o equilíbrio emocional inseparável do equilíbrio fisiológico.

Conclui-se que esta reflexão representa uma contribuição para que os enfermeiros, que atuam na área de dermatologia, utilizem como referencial as Teorias de Henderson e Kolcaba. Os conceitos propostos por essas teóricas revelam a necessidade da aquisição de competência para entender a diversidade cultural das pessoas e habilidades específicas ao desenvolvimento individualizado.

\section{REFERÊNCIAS}

1. Watson J. Enfermagem pós-moderna e futura. Lisboa(Pt): Lidel; 2002.

2. Pokomy ME. Nursing theorists of historical significance. In: Alligood MR, Marriner-Tomey A. Nursig theorists and their work. 7a ed. USA: Mosby Elsevier; 2010. p.54-68.

3. Ferin CF, Rodrigo MTL. El modelo de Virginia Henderson. In: Ferrín CF, Gómez MVN. De La teoria a la práctica: el pensamiento de Virginia Henderson em el siglo XXI. Barcelona(Es): Masson; 1998. p.33-8.

4. Brandão ES. Santos I dos, Lanzillotti RS. Nursing care to comfort people with immunobullous dermatoses: evaluation by fuzzy logic. Rev. enferm UERJ [Internet]. 2018 [cited 2018 Nov 5]; 26 e32877. DOI: https://doi.org/10.12957/reuerj.2018.32877

5. Kolcaba K. Comfort theory and practice: a vision for holistic health care and research. New York: Springer; 2008.

6. Down T. Katharine K: theory of confort. In: Tomey AM, Alligood MR. Nursing theorists and their work. 6a ed. New York: Mosby Elsevier; 2006. p. 726-42.

7. Kolcaba K. Holistic comfort: operationalizing the construct as a nurse-sensitive outcome. Adv Nurs. Sci. 1992; 15(1):1-10.

8. Sousa FAEF. Pain, the fifth vital sign. Rev. latino-am enferm. [Internet]. 2002 [cited 2018 Nov 5]; 10(3):446-7. DOI: http://dx.doi.org/10.1590/S0104-11692002000300020

9. Bottega FH, Fontana RT. Pain as the fifth vital sign: use of the assessment scale by nurses in general hospital. Texto \& contexto enferm. [Internet]. 2010 [cited 2018 Nov 5]; 19(2). DOI: http://dx.doi.org/10.1590/S0104-07072010000200009

10. Potter PA, Perry AG. Mobilidade e imobilidade. 7a ed. Rio de Janeiro: Elsevier; 2009. p.1219-78.

11. Potter PA, Perry AG. O sono. 7a ed. Rio de Janeiro: Elsevier; 2009. p.1028-49.

12. Santos I dos, Jesus PBR, Brandão ES. Oliveira EB, Silva AV. Repercussions of skin conditions in people's lives: sócio-poetizing selfimage and self-esteem. Rev. enferm UERJ [Internet]. 2014 [cited 2018 Nov 5]; 22(2):157-62. Available from: https://www.epublicacoes.uerj.br/index.php/enfermagemuerj/article/view/13545/18826

13. Goffman E. Estigma. 4a ed. Rio de Janeiro: Guanabara Koogan; 1988.

14. Braden N. Autoestima: como aprender a gostar de si mesmo. 38a ed. São Paulo: Saraiva; 2001.

15. Santos I, Brandão ES, Clos AC. Dermatology nursing: sensitive listening skills and technology for acting skincare. Rev. enferm. UERJ [Internet]. 2009 [cited 2018 Nov 5]; 17(1):124-30. Avaiable from: http://www.facenf.uerj.br/v17n1/v17n1a23.pdf 